\title{
Recola2: a one-loop matrix-element generator for BSM theories and SM effective field theory
}

\author{
Ansgar Denner, Jean-Nicolas Lang* \\ Julius-Maximilians Universität Würzburg, 97074 Würzburg, Germany \\ E-mail: jlang@physik.uni-wuerzburg.de
}

Sandro Uccirati

Università di Torino e INFN, 10125 Torino, Italy

E-mail: ucciratieto.infn.it

\begin{abstract}
We present the RECOLA2 library for the efficient generation and computation of one-loop amplitudes in Beyond-Standard-Model theories. RECOLA2 is based on RECOLA, an efficient one-loop amplitude generator for the Standard Model, and REPT1L, a newly developed tool to generate one-loop model files for RECOLA2 in a fully automated way. RECOLA2 is able to operate with non-trivial extensions of the SM, e.g. extended Higgs sectors and effective field theories. We discuss first applications to extended Higgs sectors and their renormalization.
\end{abstract}

13th International Symposium on Radiative Corrections

24-29 September, 2017

St. Gilgen, Austria

\footnotetext{
* Speaker.
} 


\section{Introduction}

One of the burning questions in particle physics concerns the precise nature of the Higgs boson. In the Standard Model (SM) the Higgs mechanism is implemented in a minimal fashion, predicting the existence of a single physical Higgs boson, but many extensions of the SM require this Higgs boson to be accompanied by additional scalar bosons in order to account for astrophysical observations. To be compatible with current measurements the additional Higgs bosons can only couple weakly to other SM particles, in particular to the gauge bosons. This constraint requires any extended Higgs sector to be (almost) aligned with the SM, giving rise to SM-like tree-level couplings between SM particles. Thus, interesting signatures are expected to arise only in the electroweak (EW) corrections of the corresponding models, and these are needed to be computed in order to distinguish a Beyond-Standard-Model (BSM) signature from the SM "background". The so-called aligned and decoupling scenarios are not restricted to extended Higgs sectors, and similar limits exist in theories with an extended gauge group or extended space-time symmetry. In order to study all these interesting extensions automated tools are required which allow for precision predictions with realistic final states including QCD and EW corrections.

\section{Recola2: A general one-loop amplitude provider}

The QCD NLO revolution a decade ago was only the beginning of a continuous improvement and development of new highly efficient algorithms for one-loop amplitudes. Today the computation of QCD and EW one-loop amplitudes in the SM is fully automated in various approaches, overcoming the deficits of the traditional Feynman-diagram approach with sophisticated posterior algebraic simplification or recursion techniques $[1,2,3,4,5]$ and new recursive algorithms without or only partially resorting to Feynman diagrams $[6,7,8,9,10]$. With RECOLA2 we use current technology and push limitations to the next level, enabling the fully automated computation of tree and one-loop amplitudes in BSM and SM effective field theories. As the successor of the RECOLA library, RECOLA2 is naturally based on the very same off-shell recursion relations [9] without referring to Feynman diagrams at any stage in the computation. At tree-level it uses Berends-Giele off-shell recursion [11] to compute tree-level amplitudes directly. At one-loop level, amplitudes $\mathscr{M}_{1}$ are first decomposed in terms of tensor coefficients $c$ and tensor integrals $T$ as

$$
\mathscr{M}_{1}=\sum_{k} c_{k, \mu_{1} \ldots} T_{k}^{\mu_{1} \ldots}
$$

The tensor coefficients, encoding the model and process dependence, are computed fully recursively by means of an extended version of the off-shell algorithm by van Hameren [12], whereas the (model-independent) results for tensor integrals are obtained from the Collier library [13, 14]. Even though being based on the same algorithmic structure RECOLA2 overcomes many shortcomings of the original RECOLA library. In summary, RECOLA2 has been generalized with respect to RECOLA in the following ways [15]:

- All hard-coded structures, in particular SM-specific pieces of the code, have been replaced with dynamic structures, and any necessary model-dependent information is retrieved from RECOLA2-specific model files. 
- The process-generation phase has been generalized to arbitrary processes involving new fields and higher $n$-point vertices. The algorithmic implementation has been improved by excessive use of recursive functions. A new feature of RECOLA2 allows to write the recursion relations as FORM code [16], permitting the reconstruction of tree and one-loop amplitudes analytically. This feature is predominantly used in the renormalization and computation of rational terms for new models (see Section 3). Finally, the process generation has been extended to deal with model files formulated in the Background-Field Method (BFM).

- The process-computation phase has been extended accordingly to deal with new couplings, Lorentz structures and higher $n$-point vertices. Most notably, new composite Lorentz structures, e.g. linked to non-renormalizable operators, are supported dynamically, making computations involving higher powers of loop momenta in Feynman rules possible. For instance, this ingredient has been used for the implementation of the $R_{\xi}$-gauge at one-loop level.

- So far RECOLA2 is restricted to theories with scalars, Dirac fermions and vector bosons. An enhanced version with the support of Majorana fermions will be developed in the future.

\section{Framework for one-loop renormalization}

In RECOLA2 the computation of renormalized one-loop amplitudes consists of the computation of the loop amplitude (2.1), in addition to rational terms of type $R_{2}$ and counterterms:

$$
\mathscr{M}_{1}^{\text {Ren }}=\sum_{c} c_{\mu_{1} \ldots} T_{c}^{\mu_{1} \ldots}+\mathscr{M}_{\mathrm{R}_{2}}+\mathscr{M}_{\mathrm{CT}} .
$$

The appearance of the extra rational terms is due to the use of dimensional regularization and the fact that we compute the tensor coefficients in exactly 4 dimensions. These remnants, emerging from the $4-D$ dimensional part of tensor coefficients and the pole part of tensor integrals, can, however, be implemented as special Feynman rules treated on equal footing with the counterterms of the theory. Thus, one step towards the evaluation of (3.1) for generic theories is to compute the rational terms of type $\mathrm{R}_{2}$ and the counterterms for each model file once and for all. The second step in our approach is to bring the Feynman rules into the right format suited for our recursion techniques, resulting in RECOLA2-specific model files. In summary, our approach towards the automated derivation of one-loop model files consists of the following 3 steps:

1) In the first step we define the model in terms of a Lagrangian with a subsequent derivation of the tree-level Feynman rules in the UFO format [17]. We use tools like FEYNRULES [18, 19] and SARAH [20] to perform this task.

2) Then, we compute the rational and counterterm vertices analytically using the newly developed tool REPT1L [15]. The main ingredient for this kind of computations is the ability of RECOLA2 to derive one-loop vertex functions analytically. This is done using the RECOLA2 process generator and FORM for the analytic reconstruction of amplitudes from the process skeletons. For more details on the computation and renormalization procedure we refer to the original reference [15]. 
3) Finally, all the (additional) Feynman rules can be mapped to the recursion kernels, resulting in a renormalized model file for RECOLA2 ready for computations of, in principle, arbitrary processes in the underlying theory.

\section{Application to extended Higgs sectors}

As a first application for REPT1L and RECOLA2 we have chosen the Higgs-singlet extension of the SM and the Two-Higgs-Doublet model. The renormalization is performed as far as possible following the on-shell renormalization of the SM, i.e. all parameters associated to fields are renormalized in the on-shell or Complex-Mass scheme [21, 22, 23]. For the gauge couplings we support the conventional schemes: The strong coupling constant can be renormalized in a fixed and variable flavour (running) scheme, whereas the electroweak coupling constant can be renormalized in the Thomson limit, at the $\mathrm{Z}$ pole, or in the Fermi scheme. The remaining independent parameters can be identified as mixing angles, which, at leading-order, describe the mixing between fields in the interaction and mass eigenbases. As their renormalization is controversial, we support various schemes encountered in the literature:

$\overline{\mathrm{MS}}$ : We support a large class of $\overline{\mathrm{MS}}$ schemes which are all defined by requiring a vanishing pole part of a suited vertex function. The actual vertex function is irrelevant as long as the parameter to be renormalized appears in the corresponding counterterm vertex. The parameter of choice can be either a mixing angle or a parameter of the Higgs potential. ${ }^{1}$ In addition, we support the renormalization in different tadpole counterterm schemes, which, however, leads to gauge-dependent results in combination with an $\overline{\mathrm{MS}}$ renormalization of the mixing angles unless the Fleischer-Jegerlehner tadpole scheme [24, 25, 26] is used. In this way the different $\overline{\mathrm{MS}}$ schemes proposed in Refs. [26, 27, 28] are obtained.

on-shell: The on-shell schemes are defined by requiring that certain mixing energies ${ }^{2}$ vanish at specific phase-space points. The procedure is gauge dependent and requires an ad hoc prescription to render the $S$-matrix gauge independent. This procedure amounts to, roughly speaking, fixing the value for the mixing angle counterterm in a particular gauge. We implemented the schemes proposed in Refs. $[25,29,30]$ using the BFM for the quantum gaugeparameter set to one and translated the results to the conventional formulation in the 't HooftFeynman gauge.

The model files together with the RECOLA2 library are publicly available under:

$$
\text { recola.hepforge.org }
$$

Each of the renormalized model files has also been derived in the Background-Field formulation of quantum field theory. This serves as a powerful complementary computation method, enabling cross checks of all the ingredients entering one-loop renormalized amplitudes (3.1).

\footnotetext{
${ }^{1}$ Since the number of independent parameters is fixed, this step requires to trade one of the mixing angles for one of the Higgs self couplings $\lambda_{i}$.

${ }^{2}$ For the considered models the condition is imposed on the neutral Higgs-boson mixing and the charged/pseudoscalar Higgs-to-Goldstone-boson mixing.
} 


\section{Summary}

We presented the RECOLA2 library for the automated generation and computation of tree and one-loop amplitudes in Beyond-Standard-model and effective field theories. The RECOLA2 library requires renormalized model files in a special format which are derived in a fully automated way using the newly developed tool REPT1L from nothing but the Feynman rules in the UFO format. We demonstrated the capabilities using the example of extended Higgs sectors. The code RECOLA2 and a set of corresponding model files are publicly available under recola.hepforge.org.

\section{References}

[1] S. Frixione, V. Hirschi, D. Pagani, H.-S. Shao and M. Zaro, JHEP 1506 (2015) 184 doi:10.1007/JHEP06(2015)184 [arXiv:1504.03446 [hep-ph]].

[2] G. Cullen et al., Eur. Phys. J. C 74 (2014) no.8, 3001 doi:10.1140/epjc/s10052-014-3001-5 [arXiv:1404.7096 [hep-ph]].

[3] M. Chiesa, N. Greiner and F. Tramontano, J. Phys. G 43 (2016) no.1, 013002 doi:10.1088/0954-3899/43/1/013002 [arXiv:1507.08579 [hep-ph]].

[4] T. Hahn, Comput. Phys. Commun. 140 (2001) 418 doi:10.1016/S0010-4655(01)00290-9 [hep-ph/0012260].

[5] T. Hahn, S. Paßehr and C. Schappacher, PoS LL 2016 (2016) 068 [J. Phys. Conf. Ser. 762 (2016) no.1, 012065] doi:10.1088/1742-6596/762/1/012065 [arXiv:1604.04611 [hep-ph]].

[6] J. Alwall et al., JHEP 1407 (2014) 079 doi:10.1007/JHEP07(2014)079 [arXiv:1405.0301 [hep-ph]].

[7] S. Kallweit, J. M. Lindert, P. Maierhöfer, S. Pozzorini and M. Schönherr, JHEP 1504 (2015) 012 doi:10.1007/JHEP04(2015)012 [arXiv:1412.5157 [hep-ph]].

[8] F. Buccioni, S. Pozzorini and M. Zoller, arXiv:1710.11452 [hep-ph].

[9] S. Actis, A. Denner, L. Hofer, A. Scharf and S. Uccirati, JHEP 1304 (2013) 037 doi:10.1007/JHEP04(2013)037 [arXiv:1211.6316 [hep-ph]].

[10] S. Actis, A. Denner, L. Hofer, J. N. Lang, A. Scharf and S. Uccirati, Comput. Phys. Commun. 214 (2017) 140 doi:10.1016/j.cpc.2017.01.004 [arXiv:1605.01090 [hep-ph]].

[11] F. A. Berends and W. T. Giele, Nucl. Phys. B 306 (1988) 759. doi:10.1016/0550-3213(88)90442-7

[12] A. van Hameren, JHEP 0907 (2009) 088 doi:10.1088/1126-6708/2009/07/088 [arXiv:0905.1005 [hep-ph]].

[13] A. Denner, S. Dittmaier and L. Hofer, PoS LL 2014 (2014) 071 [arXiv:1407.0087 [hep-ph]].

[14] A. Denner, S. Dittmaier and L. Hofer, Comput. Phys. Commun. 212 (2017) 220 doi:10.1016/j.cpc.2016.10.013 [arXiv:1604.06792 [hep-ph]].

[15] A. Denner, J. N. Lang and S. Uccirati, JHEP 1707, 087 (2017) doi:10.1007/JHEP07(2017)087 [arXiv:1705.06053 [hep-ph]].

[16] B. Ruijl, T. Ueda and J. Vermaseren, arXiv:1707.06453 [hep-ph].

[17] C. Degrande, C. Duhr, B. Fuks, D. Grellscheid, O. Mattelaer and T. Reiter, Comput. Phys. Commun. 183 (2012) 1201 doi:10.1016/j.cpc.2012.01.022 [arXiv:1108.2040 [hep-ph]]. 
[18] N. D. Christensen and C. Duhr, Comput. Phys. Commun. 180 (2009) 1614 doi:10.1016/j.cpc.2009.02.018 [arXiv:0806.4194 [hep-ph]].

[19] A. Alloul, N. D. Christensen, C. Degrande, C. Duhr and B. Fuks, Comput. Phys. Commun. 185 (2014) 2250 doi:10.1016/j.cpc.2014.04.012 [arXiv:1310.1921 [hep-ph]].

[20] F. Staub, Comput. Phys. Commun. 185 (2014) 1773 doi:10.1016/j.cpc.2014.02.018 [arXiv:1309.7223 [hep-ph]].

[21] A. Denner, S. Dittmaier, M. Roth and D. Wackeroth, Nucl. Phys. B 560 (1999) 33 doi:10.1016/S0550-3213(99)00437-X [hep-ph/9904472].

[22] A. Denner, S. Dittmaier, M. Roth and L. H. Wieders, Nucl. Phys. B 724 (2005) 247; Erratum: [Nucl. Phys. B 854 (2012) 504] doi:10.1016/j.nuclphysb.2011.09.001, 10.1016/j.nuclphysb.2005.06.033 [hep-ph/0505042].

[23] A. Denner and S. Dittmaier, Nucl. Phys. Proc. Suppl. 160 (2006) 22 doi:10.1016/j.nuclphysbps.2006.09.025 [hep-ph/0605312].

[24] J. Fleischer and F. Jegerlehner, Phys. Rev. D 23 (1981) 2001. doi:10.1103/PhysRevD.23.2001

[25] M. Krause, R. Lorenz, M. Mühlleitner, R. Santos and H. Ziesche, JHEP 1609 (2016) 143 doi:10.1007/JHEP09(2016)143 [arXiv:1605.04853 [hep-ph]].

[26] A. Denner, L. Jenniches, J. N. Lang and C. Sturm, JHEP 1609 (2016) 115 doi:10.1007/JHEP09(2016)115 [arXiv:1607.07352 [hep-ph]].

[27] A. Freitas and D. Stöckinger, Phys. Rev. D 66 (2002) 095014 doi:10.1103/PhysRevD.66.095014 [hep-ph/0205281].

[28] L. Altenkamp, S. Dittmaier and H. Rzehak, JHEP 1709 (2017) 134 doi:10.1007/JHEP09(2017)134 [arXiv:1704.02645 [hep-ph]].

[29] J. R. Espinosa and Y. Yamada, Phys. Rev. D 67 (2003) 036003 doi:10.1103/PhysRevD.67.036003 [hep-ph/0207351].

[30] F. Bojarski, G. Chalons, D. Lopez-Val and T. Robens, JHEP 1602 (2016) 147 doi:10.1007/JHEP02(2016)147 [arXiv:1511.08120 [hep-ph]]. 